\title{
Revenue Generation In Hospital Foundations: Neural Network Versus Regression Model Recommendations
}

Mary E. Malliaris, Loyola University Chicago, USA

Maria Pappas, Thorek Memorial Foundation, USA

\begin{abstract}
This paper looks at revenue amounts generated by non-profit hospital foundations throughout the US. A number of inputs, including, among others, compensation, type of support given to the hospital, type of foundation expenditures, and hospital size, were used to develop models of foundation revenue. Both neural network and regression models were developed and compared in order to see which one gave a better model and to see how they ranked the relative value of the input variables. Though the generated value of revenue for both models correlates highly with actual revenue, the neural network shows smaller error. The order of variable importance for the models is very different. Each model would have different implications for foundations in planning their next round of revenue generating events.
\end{abstract}

Keywords: hospital foundation; neural network; regression; revenue generation

\section{INTRODUCTION}

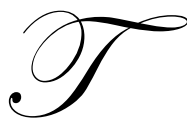

he primary focus of this research was to compare the results from two models built to determine the best indicators of revenue generation among nonprofit foundations supporting hospitals of various sizes in the United States. Hospital foundations typically contribute money to their associated hospital or the community in which the hospital is located. These contributed funds may come from investment income, gifts, or fundraising activities that the foundation coordinates.

As hospital costs soar, foundation support may become even more important in the future. Raymond found that even though health-related philanthropic contributions have doubled in the last four decades, health care costs still exceed the rate of increase in giving related to health care. A study by Pink and Leatt looked at 80 foundations throughout Canada and found that one of the factors associated with increased foundation revenue was a higher level of foundation expenses.

Foundations are faced not only with the question of how to spend their money, but also how to raise funds. Typical foundation choices for raising the money they want to give away come from investment income and from events such as galas, fun runs, memorials, and targeted themes. Since each type of event is time-consuming, it would be beneficial to know which type brings in more funds. When it comes to spending, the foundations typically spend in four areas: 1) on their associated hospital, 2) in the community, 3) for charity care, and 4) on research/education. If spending on one of these areas encourages people to give more, then that knowledge is also beneficial to a foundation.

Currently, many foundation boards make their decisions about spending based on a consensus of the board (often volunteer) that runs the foundation. The cover article of the April 2010 issue of Trustee, a publication for hospital boards, discussed the important impact on a hospital from foundation money. It mentioned the importance of joint communication between the hospital board and the foundation board so that they can emphasize the same projects. It also suggested that the two boards meet together periodically to discuss such projects. 
Though these articles agree on the importance of a foundation's contribution, they do not develop statistical models for revenue decisions. Rather, they rely on reasoned decisions consistent with a mission to make decisions about raising and spending foundation money.

This study will look at a number of hospital foundations in the U.S. and compare the results from using regression and neural network models as indicators of how their money should be handled. These models will enable us to see what factors contribute most to increased revenue.

\section{DATA}

A sample of foundations was selected from a variety of hospital sizes and locations throughout the U.S. The term "hospital foundation" describes a non-profit organization which devotes its efforts and resources to the support of a single hospital. All foundations researched are non-profit organizations, classified as 501(c)(3) and thus considered tax-exempt by the federal government. These organizations file a Form 990 annually to report their finances and revenue generating operations. These 990 filings were the basis for most of the data in this study.

Information from the Form 990s was collected from Guidestar.org (a non-profit reporting website) with financial data for 2005, a year available for all foundations. The 990 filings contain much important financial information about non-profits. In addition, the foundation's website yielded data regarding the hospital size, the foundation's board of directors, foundation staff members, fundraising campaigns and foundation events. Data not available on the internet was retrieved through direct phone contact with hospital foundations.

Data acquired for each foundation described identifying, operating, and financial characteristics of the foundation. Identifying characteristics were used to determine the regional location of each foundation and the size of the associated hospital served. Hospitals were separated into five groups according the number of licensed beds: 1) Large (400 or more), 2) Medium/Large (300-399), 3) Medium (200-299), 4) Small/Medium (100-199), and 5) Small (0-99). Table 1 shows the number of foundations included from each region and for each hospital size.

Table 1: Number Of Foundations Per Region, By Hospital Size

\begin{tabular}{|l|c|c|c|}
\hline \multicolumn{1}{|c|}{ Region } & Count & HospSize & Count \\
\hline Midwest & 50 & Large & 22 \\
\hline Northeast & 50 & Medium & 72 \\
\hline South & 40 & MedLarge & 20 \\
\hline West & 42 & Small & 25 \\
\hline & & SmallMed & 43 \\
\hline
\end{tabular}

Operating Activities describe foundation strategy for generating revenue, foundation fundraising events and leadership. Operating fields include the type of strategy followed to collect funds (primarily fundraising, investing, or mixed), types of fundraising campaigns, types of events (memorial, athletic, party, giving), Number of Board members, Board size category, Number of staff members employed, Staff size category, and Donation recipients (community, hospital, charity, and research).

Financial fields include Annual contributions, Annual revenue, Net assets, Annual expenses, Board compensation cost, Staff compensation cost, and Fundraising compensation cost.

Hospital foundations do not all spend money in similar ways. Some support their boards, their staff, and/or fundraisers while others rely on volunteer support. The effect of CEO wages on foundation performance has been studied, as well for as executive directors and donors.

In addition, foundations raise money in various ways and spend their money on a variety of projects. For simplicity, we have divided the ways foundations raise money into four types of categories: 1) party galas, 2) athletic events, 3) giving drives (such as radio-thons or auctions), and 4) memorial events. The recipients of foundation money have also been categorized into four areas: 1) the associated hospital, 2) the community in which 
the hospital is located, 3) charity care, and 4) research or education projects. The money spent on boards, staff and fundraisers were entered as dollar amounts, while the ways money was raised or spent was entered as one if money was raised or spent that way, and zero otherwise. Some foundations raise and spend money in multiple ways.

\section{FOUNDATION STRUCTURE}

The governing body of a foundation is the board of directors. They oversee the organization's assets and make decisions about distributions. They represent the interests of both the community and the associated hospital. In some cases, boards contain members who represent a corporate partner or annual event sponsor. Foundation boards may be paid or voluntary. The decisions about allocation of funds are reached when this board meets to discuss how they view the foundation spending as supporting the foundation goals. Sometimes, there is no board and in such a case, the foundation staff assumes greater responsibility.

The foundation staff provides full-time support for the board. Staff often focus on the daily task of soliciting funds from new and past donors. Of the foundations in this study, only seven reported no staff. The absence of staff often indicates that the board or some hospital employees assume these responsibilities when necessary. Typical staff position titles might be Executive Director, Director of Development, or Events Manager. Staff can also be paid or voluntary.

Some foundations employ professional fundraisers to identify and motivate donors, or grant writers to ensure proper allocation and use of funds. Foundations without the professionals rely on volunteers or auxiliaries to execute fundraising events.

\section{MODELS}

The current literature mentions collaboration between hospital and foundation boards, and discussion about projects to take on, but the arguments used are based on aligning spending with mission, rather than based on the outcome of a statistical model. This paper proposes to use two models that have been shown to be popular in other areas and apply them to decision making for foundations.

The two methodologies used to construct models of hospital foundation revenue were a neural network model and a multiple regression model. Each used the same inputs and the same target (Revenue). We wanted to compare not only the accuracy of the two models, but also the order of variable importance. Foundation money and the time available for board and staff involvement are always limited. So, it is of interest to see which variables each model would recommend as contributing more to generation of revenue.

The fields used to build the neural network and regression models for this study were restricted to the following fields: number of beds in the associated hospital, amount of contributions for the year, total expenses for the year, amount spent on compensation for board members, compensation for staff, and compensation for fundraising, net assets, and eight 0/1 fields reflecting the type of fundraisers held (memorial, athletic event, party, giving drive) and project area the foundation supports (hospital, charity, research, community). The dependent variable used was the amount of revenue brought in for the year.

Both models were built and run using the SPSS package Clementine. Clementine is a data mining tool with drag and drop functionality. Model pieces are represented by nodes that perform one function each. Nodes are used to read in the data, process the data, and display results from the completed models. The data was read in and then sent through a type node. The type node stores the data type for each variable and its use in the model (as input or output). Each model is attached to the type node. After a model is executed, it generates a trained model which is indicated by a gold nugget. These nuggets can be attached to the type node in order to analyze and graph their output.

SPSS generated models also produce a graph of variable importance. This graph shows the importance each model gives to the various inputs used for the projection of the revenue value. Variable importance values in Clementine are relative. The sum of the values for all input variables in each model is 1.0. Variable importance does 
not relate to model accuracy. It just relates to the importance of each variable in making a prediction, not whether or not the prediction is correct. A variable's importance to the dependent variable value is calculated by looking at how much the dependent variable changes when the lowest and highest values of the variable are fed through the model and all other variable values are held constant.

Several configurations of networks were tried with varying numbers of hidden layer nodes. Since there were 15 inputs, the configurations tested included a smaller number of hidden layer nodes (7), and equal number (15), and a larger number (30). Each of these configurations had similar accuracies (99.5, 99.57, 99.573 respectively). The training time increased as the number of hidden nodes increased ( $1 \mathrm{~min} 27 \mathrm{sec}, 1 \mathrm{~min} 31 \mathrm{sec}$, and $2 \mathrm{~min} 13 \mathrm{sec}$ respectively). All networks had the same three top variables. The remaining variables, all of importance less than .05, had extremely small differences in importance value. Lastly, the neural network configuration with 15 hidden layer inputs was tried with various sorts on the input variables (alphabetical, by type of data, randomly). These again all showed the same top three variables with the remaining below .05 in impact.

The final neural network model was set up with 15 inputs, one hidden layer of 15 nodes and one output (Revenue). The regression model used the same 15 inputs and one output. The results for model accuracies, generated by the analysis node, are shown in Table 2. Here we see that the error values reported for the neural network model are better than for the regression model and the correlation values for both models indicate the ability to model this data set well. Both models have a high linear correlation with the actual target.

Table 2: Model Accuracy Results

\begin{tabular}{|l|c|c|}
\hline & Reg & NeuNet \\
\hline Minimum Error & -2.206 & -2.42 \\
\hline Maximum Error & 18.099 & 4.165 \\
\hline Mean Absolute Error & 1.709 & 0.447 \\
\hline Standard Deviation & 3.37 & 0.718 \\
\hline Linear Correlation & 0.96 & 0.998 \\
\hline
\end{tabular}

Graphing each model versus the actual value of revenue also shows the indication of a high correlation between the actual value of revenue and the model-generated value. Figure 1 shows the regression model results and Figure 2 shows the results for the neural network model.

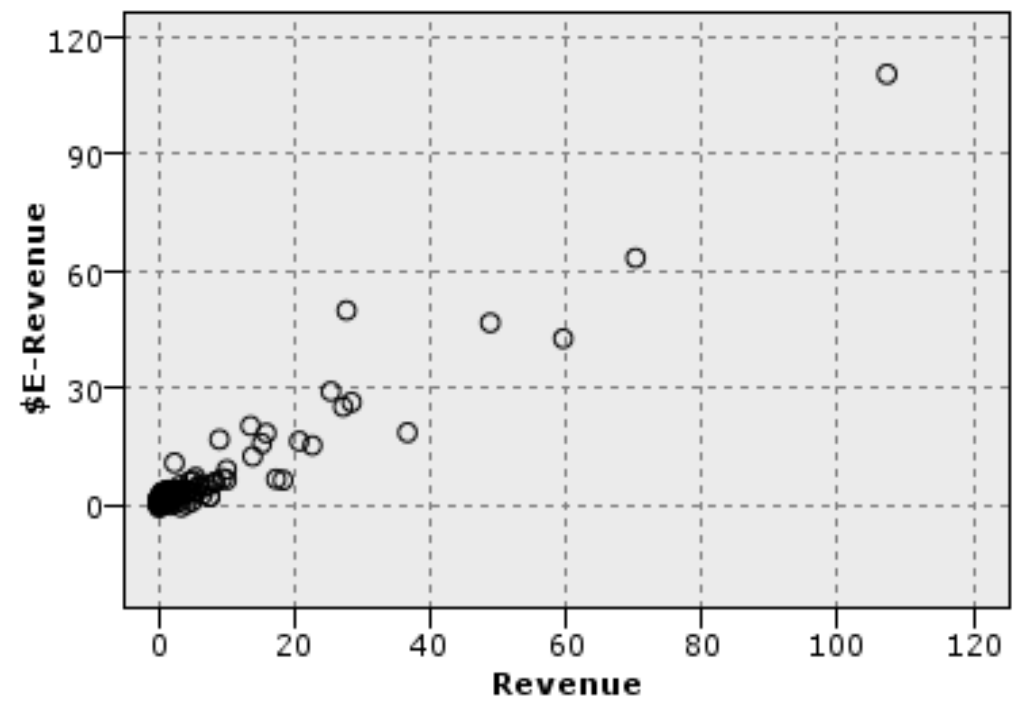

Figure 1: Revenue vs. Regression Projection 


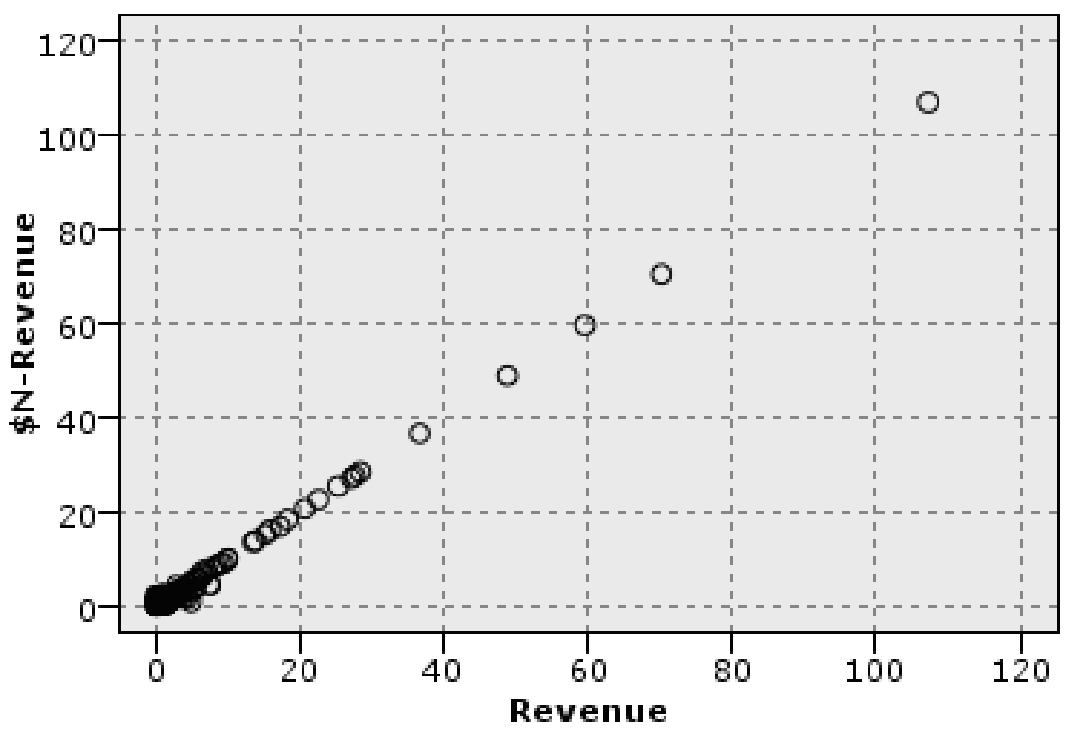

Figure 2: Revenue vs. Neural Network Projection

The relative variable importance for each of the models is displayed next. Figure 3 shows the results from the regression model. By far the most important variable for the regression model is the amount reported for expenses. The relative importance of this variable is 0.7 out of 1.0. The importance here of expenses agrees with the conclusions of the Pink and Leatt study. All the other variables in the model have relative importance values below 0.1. The second and third variables in relative importance are Hospital (a variable showing where money is allocated) and Giving (an indication of the most profitable type of fund-raising event).

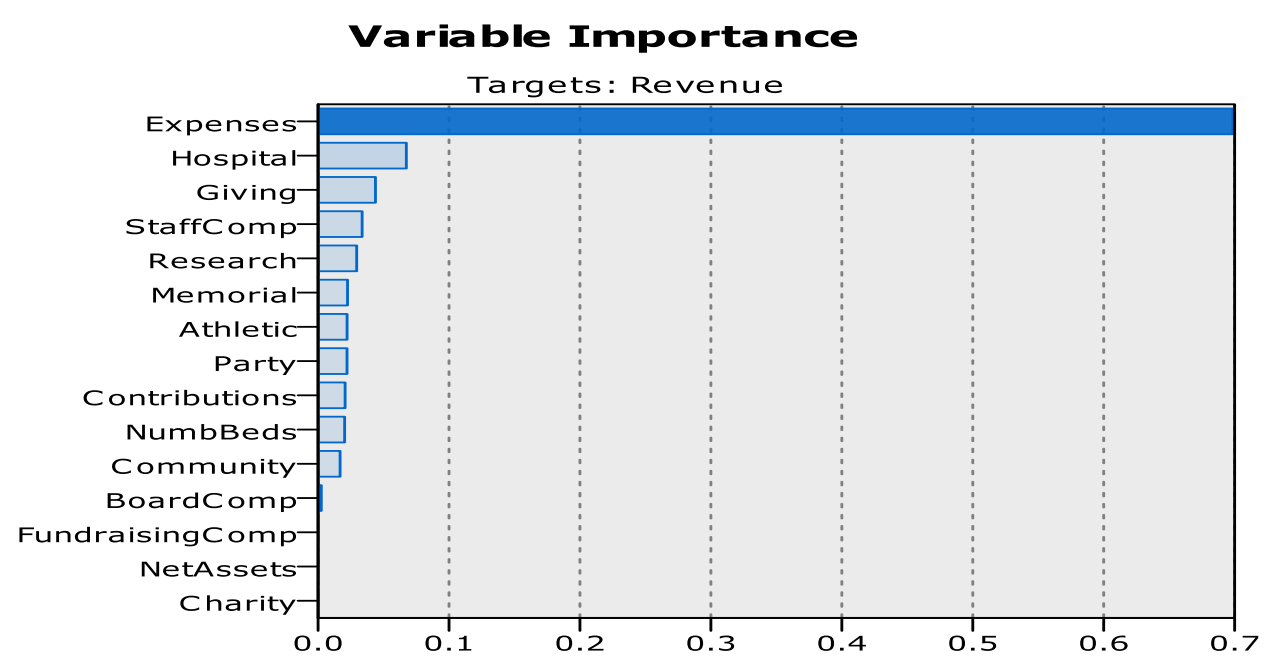

Figure 3: Rel. importance of input variables, regression model 


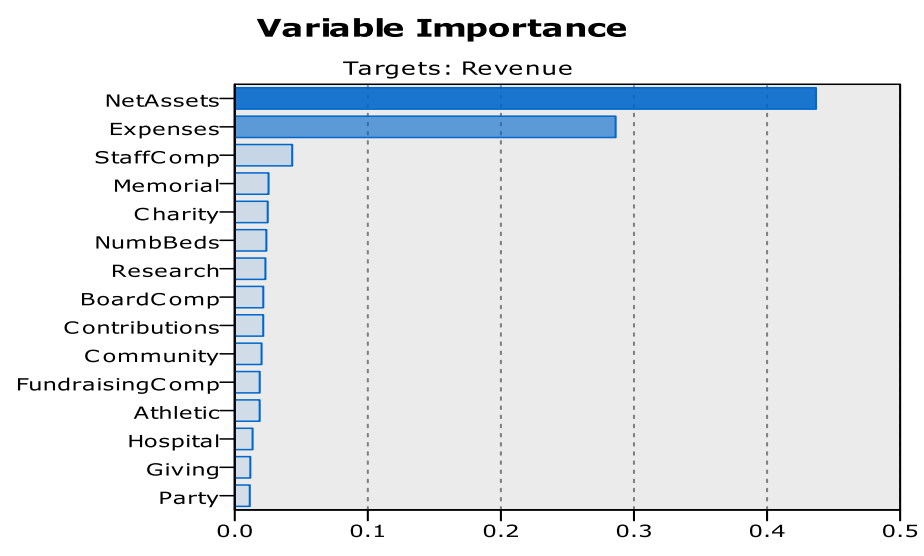

Figure 4: Rel. importance of input variables, neural network model

The neural network model rankings of variable importance are shown in Figure 4. Though expenses are high on the list, the variable with the highest relative importance in determining revenue for the neural network is the variable Net Assets with a relative importance value of .44. This is followed by Expenses, then Staff Compensation.

Table 3 gives the values of the variable importance for each of the variables and the absolute value of the difference between the two. The table is sorted by absolute difference. The correlation between the two columns of importance is .4595 , indicating that there is a lack of agreement between the relative variable importance assigned by each of the two models.

These two models give very different pictures of variable importance in the generation of revenue. Net Assets is the most important variable to the neural net while it is the least important to the regression model. Expenses rank highest on the regression model and second for the neural net, though the amount of importance given to the variable varies by .4125. For future activities, a foundation is always interested in the best way to get and give money. The regression model ranks the best way to give money as "hospital". This choice would give the money to the associated hospital in an unrestricted way to be used for eqiupment or programs.

Table 3: Comparative Variable Importance Values for Models

\begin{tabular}{|l|c|c|c|}
\hline \multicolumn{1}{|c|}{ Variable } & NN & Reg & Abs Dif \\
\hline NetAssets & 0.437 & 0.000 & 0.437 \\
\hline Expenses & 0.286 & 0.699 & 0.413 \\
\hline Hospital & 0.013 & 0.067 & 0.054 \\
\hline Giving & 0.012 & 0.044 & 0.032 \\
\hline Charity & 0.025 & 0.000 & 0.025 \\
\hline BdComp & 0.021 & 0.003 & 0.019 \\
\hline FndComp & 0.019 & 0.000 & 0.019 \\
\hline Party & 0.011 & 0.022 & 0.011 \\
\hline StfComp & 0.043 & 0.034 & 0.010 \\
\hline Research & 0.023 & 0.030 & 0.007 \\
\hline Beds & 0.024 & 0.020 & 0.004 \\
\hline Athletic & 0.019 & 0.022 & 0.003 \\
\hline Community & 0.020 & 0.017 & 0.003 \\
\hline Memorial & 0.025 & 0.023 & 0.003 \\
\hline Contrib & 0.021 & 0.021 & 0.001 \\
\hline
\end{tabular}


The regression model gives greatest importance to "giving events" such as auctions and radio-thons for the way for the foundation to raise money. In contrast, following the neural network recommendation, the foundation would give money first for "charity care". This encompasses individuals who receive care from the hospital but do not have the ability to pay for services. The neural network gives most importance to "memorial programs" for raising money. These involve personal memorial, remembrance, or naming opportunities.

Which types of actions do each of the models consider least important for generating revenue? The regression model assigns the least importance to giving money for "charity care", the direct opposite of the neural network model. It identifies "party", in other words, gala events or dances, as the least beneficial way to increase revenue. The neural network gives the least importance to giving to the "hospital", again the opposite choice of the regression model. It, however, agrees with the regression model in identifying "party" as of lowest importance in revenue generation.

In the matter of compensation paid to the board, the staff, and professional fundraisers, the models also differ. Foundations are not allowed to pay bonuses to staff or Board members for soliciting contributions, so none of the expenses reported as fundraising compensation are funds paid to foundation or hospital employees for achieving monetary goals or quotas. The regression model gives importance above .01 only to staff compensation. The neural network, however, has importance measures of $.02, .04$, and .018 respectively to the roles of board, staff, and fundraisers. Following the regression recommendation, we would pay only the staff. Following the neural network recommendation, we would pay all three categories. Foundations which report staff compensation may benefit from fundraising efficiencies with higher average assets, contributions and revenues. Cohen reports that most non-profits having contributions over a million have fundraising staff. These increases offset the additional expenses incurred. Frederick and Rooney find that nonprofits are more involved in raising money when they have fundraising staff. Foundations in this data set that reported fundraising compensation had the greatest increases in assets, contributions, revenues and expenses. However, the average cost of fundraising compensation was more than double those of staff and board compensation. So, although fundraising compensation results in financial increases, it is the least cost effective form of compensation. In terms of foundation expansion, it may be wise to invest in board or staff compensation first, which both cost less and produce relatively similar financial increases.

Thus, two models, both appearing to do well on the overall measures of model success, give the foundations varying strategies to follow in their revenue generation.

\section{CONCLUSIONS}

Foundations supporting hospitals follow a variety of methodologies in order to raise revenue. To attract donations effectively and efficiently, some foundations compensate staff members, board members or employ professional fundraisers. These trained professional fundraisers are proficient in many aspects of donations, especially in identifying potential donors and cultivating foundation/donor relationships. To further acquire funds, many foundations hold events such as an annual campaign or a gala evening. While these gain visibility for the foundation and the hospital it serves, they also require extensive time and expense to conduct. This often means the employment of staff. Foundations with staff may increase operating costs due to fixed salaries. Overhead is further increased by the costs of fundraising efforts. However, these costs may be worthwhile for the foundation if the staff can effectively solicit donations which exceed total expenses, providing a positive return.

This study examined a sample of hospital foundations of various sizes and in different regions of the U.S. and then compared the results of two models - neural network and regression - used to pattern the generation of revenue. Financial reports from their operations were used to gather data about input variables that might affect their bottom line. These two models suggest different paths for the foundation wishing to maximize revenue with minimum effort. The regression model suggests focusing on fundraising events with activities that directly raise money, such as auctions and radio-thons. The money raised should be spent first on the associated hospital. The neural network model suggests spending the money on charity care and raising it through programs involving personal memorials or naming opportunities. Since foundations' actions are very public; they become known for ways they raise and spend their monies. Although both models have a high correlation of predicted revenue with actual revenue, the neural network model has a lower associated error. This suggests the neural network model recommendations may be better ones to follow. 


\section{ACKNOWLEDGEMENTS}

This work was supported in part by a grant from the Thorek Memorial Foundation. We thank Tom Whitaker, graduate assistant, for his excellent work in the collection and organization of the data.

\section{AUTHOR INFORMATION}

Mary Malliaris is Associate Professor and Chair of the Information Systems \& Operations Management Department in the School of Business Administration at Loyola University Chicago. Her research and teaching interests are in data mining, statistics, financial modeling and health care informatics. She has published in The Journal of Banking and Finance, The European Journal of Finance, The American Journal of Business Education, and Neural Computing and Applications, among others and is currently production editor for The Journal of Economic Asymmetries.

Maria Pappas has been elected to her fourth term as Treasurer of Cook County, the nation's second most populous. A psychologist and a lawyer, Pappas served two terms as a county Commissioner, gaining expertise in budgets and administration. Since becoming Treasurer in 1998, she has streamlined - trimming staff by more than 50 percent - while adding services, cutting dependence on taxes and increasing interest yields on County accounts. She believes in technology, continually updating systems to collect more than $\$ 11$ billion in property taxes yearly in two installments from 1.8 million parcels and distribute the revenue to 1,600 taxing agencies.

\section{REFERENCES}

1. Raymond, S. The Role of Hospital Foundations in Health Care Philanthropy and Finance" (Part 1), 10/14/05: OnPhilanthropy.com (Retrieved October 10)

2. Pink, G. \& P. Leatt, Fund-raising by Hospital Foundations, Nonprofit management and Leadership, 2006, 1(4), 313-327.

3. Serb, C., The Business of Philanthropy, Trustee, April 2010, 63(4): 8-12.

4. Froelich, K., T. Knoepfle \& T. Pollak, Financial Measures in Nonprofit Organization Research: Comparing IRS 990 Return and Audited Financial Statement Data. Nonprofit and Voluntary Sector Quarterly, 2000, 29(2), 232-254.

5. Gordon, T., S. Khumawala, M. Kraut \& J. Meade, The Quality and Reliability of Form 990 Data: Are Users Being Misled, Academy of Accounting and Financial Studies Journal, 2007, 11.

6. Preyra, C. \& G. Pink, Balancing incentives in the compensation contracts of nonprofit hospital CEOs, Journal of Health Economics, 2001, 20(4), 509-525.

7. Brickley, J. \& R. L. Van Horn, Managerial Incentives in Nonprofit Organizations: Evidence from Hospitals. The Journal of Law and Economics, 2000, 45(April).

8. Ruhm, C. \& Carey Borkoski, Compensation in the Nonprofit Sector, J. Human Resources, 2003, 38(4):992-1021.

9. Eldenburg, L. \& R. Krishnan, Public versus private governance: a study of incentives and operational performance. Journal of Accounting and Economics, 2003, 35(3), 377-404.

10. Hallock, K. Compensation in nonprofit organizations, Research in Personnel and Human Resources Management, 2000, 19, 243-294.

11. O'Regan, K. \& S. Oster, Does the Structure and Composition of the Board Matter? The Case of Nonprofit Organizations, J. of Law, Economics, and Organization, 2000, 21(1), 205-227.

12. Callen, J., A. Klein, \& D. Tinkelman, Board Composition, Committees, and Organizational Efficiency: The Case of Nonprofits. Nonprofit and Voluntary Sector Qtrly, 2003, 32(4), 493-520.

13. Cohen, T. Most nonprofits lack fulltime fundraisers, Philanthropy Journal, Jul 30, 2004, www.onphilanthropy.com/site/News2?id=7233\&page=NewsArticle.

14. Frederick, H. \& Rooney, P. Giving and Fundraising Research: Paying for Overhead, The Center on Philanthropy at Indiana University (Retrieved October 12, 2007 via Google). 